\title{
Observed frequency-independent torque in flagellar bacterial motors optimizes space exploration
}

\author{
Mario E. Di Salvo and C. A. Condat \\ IFEG-CONICET and FaMAF, Universidad Nacional de Córdoba, 5000 Córdoba, Argentina \\ (Received 20 April 2012; revised manuscript received 29 June 2012; published 17 December 2012)
}

\begin{abstract}
A surprising feature of many bacterial motors is the apparently conserved form of their torque-frequency relation. Experiments indicate that the torque provided by the bacterial rotary motor is approximately constant over a large range of angular speeds. This is observed in both monotrichous and peritrichous bacteria, independently of whether they are propelled by a proton flux or by a $\mathrm{Na}^{+}$ion flux. If the relation between angular speed $\omega$ and swimming speed is linear, a $\omega$-independent torque implies that the power spent in active motion is proportional to the instantaneous bacterial speed. Using realistic values of the relevant parameters, we show that a constant torque maximizes the volume of the region explored by a bacterium in a resource-depleted medium. Given that nutrients in the ocean are often concentrated in separate, ephemeral patches, we propose that the observed constancy of the torque may be a trait evolved to maximize bacterial survival in the ocean.
\end{abstract}

DOI: 10.1103/PhysRevE.86.061907

PACS number(s): 87.17.Jj, 87.17.Aa, 05.40.Fb

\section{INTRODUCTION}

Oceans are environments that support a diversity of microbial life, even if the amount of dissolved organic matter is extremely low in marine habitats [1]. Oligotrophic bacteria have adapted to grow at these low nutrient concentrations, undergoing starvation periods and exploring their surroundings in search of more favorable environments. Nutrients in the oceans are often present in the form of micropatches, point sources that are available within limited time and space [2-4]. Motile bacteria swim in order to reach these nutrient sources needed to replenish their energetic stores. Since locomotion requires the expenditure of a substantial portion of their energy, these biological entities must have evolved to survive in hostile environmental conditions, learning to carry out their locomotive functions efficiently. It has long been recognized that there are various starvation-survival activity patterns that bacteria utilize when they are not in the presence of energy-yielding substrates [5]. In this connection, the existence of a lower bacterial size limit for useful locomotion is also known [6,7].

Motile bacteria swim by rotating thin helical filaments called flagella. Each flagellum is driven by a rotary motor, which is powered by the flow of ions across the cytoplasmic membrane. The influx of ions $\left(\mathrm{H}^{+}\right.$or $\left.\mathrm{Na}^{+}\right)$is due to an electrochemical gradient and generates a torque that rotates the flagellar motor $[8,9]$. More than 40 proteins are required for the assembly and rotation of each motor. Despite their complexity, bacterial flagellar motors of different species are similar in structure $[10,11]$. One of the most important measurable characteristics of a flagellar motor is its torque vs speed relationship. It was found experimentally for various bacteria that the torque remains approximately constant for a wide range of speeds, but decreases almost linearly at high rotation speeds exhibiting a kneelike feature [12-14]. It is of current interest to understand and model this behavior, which seems to be widespread in both $\mathrm{H}^{+}$- and $\mathrm{Na}^{+}$-driven flagellar motors $[15,16]$. Of course, since the bacterial world is hugely varied and complex, other forms of the torque-speed relationship may be found, but our current understanding of the subject suggests that at least a large class of bacteria should exhibit a roughly constant torque at low speeds.
Magariyama and coworkers measured simultaneously the swimming speed $v$ and the flagellar rotation rate $\omega$ of the monoflagellated bacterium Vibrio alginolyticus and the swimming speed and the flagellar-bundle rotation rate of Salmonella typhimurium, which has peritrichous flagella $[17,18]$. In both cases they found a roughly linear relation between these two quantities $(v \approx \alpha \omega)$. If we accept the proportionality between $v$ and $\omega$, and remember that the power delivered by the bacterial motor is $\Pi=\omega M$, where $M$ is the torque generated by the motor, then, in the $\omega$ region below the knee, the power $\Pi$ must be proportional to the speed $v$. Since all studied flagellar motors have a similar structure, it is reasonable to assume that all exhibit the same torque-speed relationship. In this paper we present a possible explanation of why molecular motors have evolved in such a way that their torque is rotation-rate independent over a wide range of $\omega$ : such dependence would optimize space exploration in a resource-depleted medium, such as the ocean, where bacteria initially evolved.

Recently, we studied the interplay between energetics and dynamics in bacterial motility [19]. We used a generalization of the model for energy storage, conversion, and consumption introduced by Schweitzer, Ebeling, and Tilch (SET) [20,21]. This model has been used, among other applications, to investigate the transport properties of active particles $[6,22]$ and, very recently, to interpret how nutrient concentration affects the speed distribution of the bacterium Salmonella typhimurium [23]. In Ref. [19] we found approximate analytical solutions under a number of reasonable assumptions. In particular, the separation of time scales between slow energy storage and fast swimming speed variations leads naturally to a quasistatic approximation. A long time scale (typically a few hours) characterizes the filling and emptying of the energetic stores [24,25], while a much shorter time scale (fractions of a second) characterizes speed variations [26]. The consistency and simplicity of the SET model, and the favorable comparison of the predictions of Refs. $[19,23]$ with experimental data suggest that this model is a useful tool to investigate the relation between bacterial dynamics and the microorganism energetic budget.

This paper is organized as follows. In Sec. II we review the model of Ref. [20] and the simplifications introduced in Ref. [19]. In Sec. III we discuss the special case of a bacterium swimming in a nutrient-depleted medium with the objective 
of finding the optimal exponent of the conversion function of internal into kinetic energy. Section IV presents analytical and simulation results for the dependence of the volume of the explored region with that exponent and with the bacterial size, assuming a run-and-tumble strategy. Finally, in Sec. V we summarize our results, further discuss some aspects of the problem and make a testable prediction.

\section{THE MODEL}

In the SET model [20], the velocity $\vec{v}$ of an active particle of mass $m$ is given by the modified Langevin equation,

$$
m \frac{d \vec{v}}{d t}=-\gamma \vec{v}+\frac{k(v)}{v^{2}} E(t) \vec{v}+\vec{F}(t) .
$$

Here $\gamma$ is the friction coefficient and $\vec{F}(t)$ is a stochastic force. An equation for the stored energy $E(t)$ is built on the assumption that the microorganism takes energy from the medium at a rate $q$, converts it into kinetic energy at a rate $k(v) E(t)$, and dissipates it, through metabolism and mechanical inefficiency, at a rate $c E(t)$. We can thus write

$$
\frac{d E(t)}{d t}=q-[c+k(v)] E(t) .
$$

This equation may break down at very low values of $E(t)$, for which the bacterium may stop moving or reduce its metabolic expenditure in order to prolong its life. The set of Eqs. (1) and (2) were simplified in Ref. [19] by assuming that (i) Eq. (1) is valid for the speed (we use it only to describe the run phase; the tumbles will be introduced later in the description), and (ii) the effect of thermal noise on speed is negligible. We note that the effect of Brownian noise on directional changes may be important, especially for the smallest bacteria, whose fast motion is likely to have arisen from the need to beat directional fluctuations [27], but it is not relevant for the present analysis. Rotational diffusion generates slightly curved bacterial paths and, as a consequence, the overall trajectory of a run-and-tumble bacterium will be a random walk formed by slightly bent segments instead of straight steps. With the preceding assumptions, Eq. (1) is transformed into a scalar, deterministic equation. We further assumed that the rate of energy conversion $k(v)$ has a power-law dependence on the speed $k(v)=d_{\xi} v^{\xi}$. This keeps the problem relatively simple while allowing us to generate a reasonably general family of models. Physics sets some constraints on the form of $k(v)$, which will be discussed later.

The small mass of the bacterium and the relatively slow variation in the stored energy, which occurs typically over times of the order of the minutes or hours, justify a separation between a fast variable $v(t)$ and a slow variable $E(t)$. Using this quasistatic (QS) approximation, we obtained a separate equation for the QS value of the stored energy $E_{Q}(t)$,

$$
\frac{d E_{Q}(t)}{d t}=q-d_{\xi}^{2 \psi} \gamma^{-\xi \psi} E_{Q}(t)^{2 \psi}-c E_{Q}(t)
$$

where $\psi=(2-\xi)^{-1}$ and $\xi \neq 2$. [Note a typographical error in the second term of the right-hand side of Eq. (11) in Ref. [19]]. This QS approximation was further validated by a comparison with the numerical solutions of the exact equations [19]. For each value of $E(t)$, the fast variable $v(t)$ rapidly reaches its quasiequilibrium value:

$$
v_{Q}(t)=\left[\frac{d_{\xi} E_{Q}(t)}{\gamma}\right]^{\psi} .
$$

\section{STARVING BACTERIUM}

If the bacterium lives in the ocean, a normally oligotrophic medium where organic matter is mostly distributed in separate patches, it must explore its neighborhood to find nutrients. We will assume that bacterial evolution has selected those parameters that maximize space exploration in a nutrientdepleted environment in order to increase the probability of finding energy supplies. A particularly important parameter is the exponent $\xi$. We will search for the optimal value of this parameter, i.e., the value that maximizes space exploration subject to the constraints of physical law.

We start by setting $q=0$ in Eq. (3). The resulting equation can be integrated, yielding an explicit expression for the remaining stored bacterial energy in a depleted medium as a function of time,

$$
E_{Q}(t)=\left[\left(B_{\xi}+E_{0}^{1-2 \psi}\right) e^{-c(1-2 \psi) t}-B_{\xi}\right]^{\frac{1}{1-2 \psi}},
$$

with $E_{0}$ being the stored energy at time $t=0$ and $B_{\xi}=$ $c^{-1} d_{\xi}^{2 \psi} \gamma^{1-2 \psi}$. We can now express $v_{Q}(t)$ in terms of the initial conditions, $E_{0}$ and $v_{0}$, assuming that $v_{Q}(t=0)=v_{0}$ and solving for $d_{\xi}$. Introducing the relative bacterial energy $Z(t)=E_{Q}(t) / E_{0}$, the quasiequilibrium speed $v_{Q}(t)$ thus reads

$$
v_{Q}(t)=v_{0} Z(t)^{\psi}
$$

The speed goes smoothly to zero as $t \rightarrow \infty$. Of course, the bacterium will either die or stop completely when the available energy goes below a threshold $E_{+}$. The slowdown of bacterial motion in media with low nutrient concentrations has been observed recently [23].

A starving bacterium using a run-and-tumble strategy will effectively perform a random walk with a decreasing step size. The size of the region explored by the bacterium can be estimated by calculating the mean square displacement $\left\langle r^{2}(t)\right\rangle$, which can be found using the equation [19],

$$
\left\langle r^{2}(t)\right\rangle=\frac{1}{\theta} \int_{0}^{t}\left[\int_{t^{\prime}}^{t^{\prime}+\theta} v\left(t^{\prime \prime}\right) d t^{\prime \prime}\right]^{2} d t^{\prime},
$$

where $\theta$ is the run duration, which we assume to be constant. Since we have an analytical expression for the time dependence of the speed, we can evaluate numerically $\left\langle r^{2}(t)\right\rangle$ and determine its dependence with the energy conversion rate. We can thus quantify the size of the region that a bacterium may explore in a nutrient-depleted medium by writing its radius as $R=\left\langle r^{2}(\infty)\right\rangle^{1 / 2}$. In all the calculations we take the initial stored energy density to be the same, $9.39 \times 10^{8} \mathrm{erg} / \mathrm{cm}^{3}$. This value is obtained if we follow Mitchell and assume that $1 \%$ of the cell volume is energy reserve in the form of glucose at the maximum density and that all the energy goes to drive the motors, with the efficiency of the propulsion system being $1 \%$ [27]. This last assumption also fixes the value of $c$. The friction coefficient $\gamma$ can be easily calculated by assuming that the bacterium is spherical, with radius $a$, and writing $\gamma=6 \pi \eta a$, where $\eta$ is the fluid viscosity. 

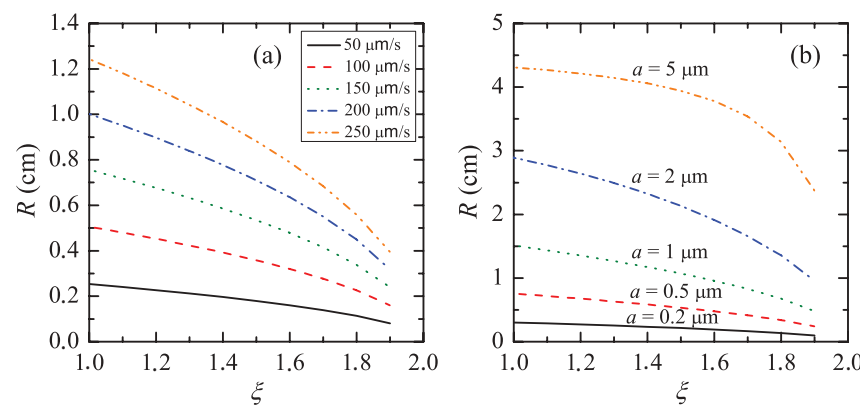

FIG. 1. (Color online) Radius of the region explored by a bacterium as a function of the power transfer exponent $\xi$. In all cases the bacterium departs with a full energy storage, corresponding to a stored energy density of $9.39 \times 10^{8} \mathrm{erg} / \mathrm{cm}^{3}$. (a) The bacterial radius is $a=0.5 \mu \mathrm{m}$ and the initial speeds are indicated in the figure. Here $E_{0}=4.91 \times 10^{-4} \mathrm{erg}, \gamma=9.42 \times 10^{-6} \mathrm{~g} / \mathrm{s}$, and $c=$ $1.94 \times 10^{-4} \mathrm{~s}^{-1}$. (b) The initial speed is now $150 \mu \mathrm{m} / \mathrm{s}$ and the bacterial radii are specified in the figure. In all cases the size of the explored region decreases with $\xi$.

\section{RESULTS}

Figure 1 shows the radius of the explored region as a function of $\xi$. In panel (a) we vary the initial speed, which is assumed to be the stationary speed consistent with the level of stored energy, while in panel (b) we vary the bacterial size. It is clear that bigger and more energetic bacteria explore larger regions, but we also observe that the sizes of these regions decrease monotonically with increasing $\xi$.

A crucial point is to note that since $\Pi=\omega M(\omega)$, and we are assuming that the rotational speed $\omega$ is proportional to the translational speed $v$, the power-law dependence of $\Pi$ implies that $k \omega^{\xi} \sim \omega M(\omega)$, that is, $M(\omega) \sim \omega^{\xi-1}$. Therefore, $\xi<1$ is impossible, because it would imply an unphysically strong torque at low $\omega$. Since $R$ is always a monotonically decaying function of $\xi$, the optimal value of this parameter must therefore be close to unity. Moreover, because the explored volume scales with the cube of $R$, the benefits of choosing the suitable strategy $(\xi \approx 1)$ are substantial in all cases. This can be seen from Fig. 2(a), where we plotted the maximum volume visited by the bacterium as a function of its size for various values of the exponent $\xi$. Note also that the relative increase between the explored volumes for different values of the exponent $\xi$ is larger for small bacteria. We remark that these volumes must always be considered as upper bounds, since the bacterium is likely to stop before exhausting its energetic stores.

Figure 2(b) shows the explored volume normalized to the volume of the bacteria doing the exploring. Since bacteria explore to get nutrients, this plot tells us how successful the exploration will be for a given size bacterium. We see that even if $\xi=1$ is always the exponent that allows for the most extensive space exploration, the competitive advantage of having a value of $\xi$ close to one is much bigger for small bacteria. This competitive advantage is independent of size for $a \lesssim 1.5 \mu \mathrm{m}$, but comes down for bacteria whose radius is larger than this value.

To build Figs. 1 and 2 we used experimentally reasonable values, but to further analyze the problem, it is convenient to rewrite the equation for the stored energy in a nutrient-depleted
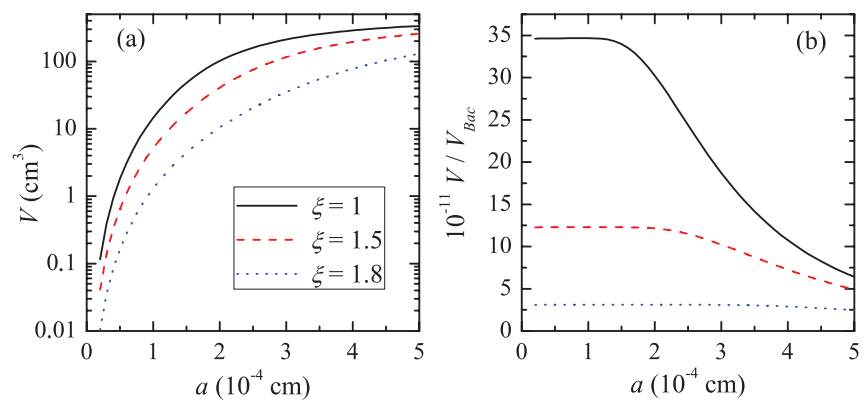

FIG. 2. (Color online) (a) Volume of the region explored by a bacterium as a function of bacterial size for various values of the power transfer exponent $\xi<2$. Parameters were chosen so that in all cases the initial speed, which we took to be equal to the corresponding run speed, is $150 \mu \mathrm{m} / \mathrm{s}$. (b) Explored volume for the bacteria in (a) normalized to the corresponding bacterial volume, $V_{\mathrm{Bac}}$.

medium, Eq. (3), in its dimensionless form. Calling $\tau \equiv c t$, we find

$$
\frac{d Z(t)}{d \tau}=-\frac{\gamma v_{0}^{2}}{c E_{0}} Z(t)^{2 \psi}-Z(t) .
$$

Note that since the energy decreases as time goes by, $Z<$ $1, \forall t>0$. The coefficient of the first term on the right-hand side can be rewritten as

$$
\frac{\gamma v_{0}^{2}}{c E_{0}}=\frac{d_{\xi} v_{0}^{\xi}}{c} \equiv \Gamma_{0} .
$$

If $\Gamma_{0} \ll 1$, this term can be neglected and the emptying of the energy depot will be exponential. The coefficient $\Gamma_{0}$ represents the initial ratio between the power spent on active motion and that spent on metabolism. As remarked by various authors, bacteria spend most of their internal energy for metabolism $[7,27]$; taking into account the inefficiencies of the energy conversion in the propulsion system, a reasonable estimate is $\Gamma_{0} \sim 10^{-2}$ and thus, at times not too long, the first term can indeed be neglected. However, recent experimental evidence suggests that in some occasions the energy spent in both processes may be comparable [23].

To complete the analysis, we must investigate the case $\xi \geqslant$ 2. As we showed before [19], in this case there is a lower threshold $q_{c}^{(\xi)}$ in the rate of nutrient uptake required to keep the microorganism moving:

$$
q_{c}^{(\xi)}=\frac{\gamma \xi}{\xi-2}\left[\frac{c(\xi-2)}{2 d_{\xi}}\right]^{\frac{2}{\xi}}, \quad \xi \geqslant 2 .
$$

If the microorganism finds itself in a large region where the nutrient concentration is so low that $q<q_{c}^{(\xi)}$, all organized motion must eventually stop. This is the case for a bacterium in a nutrient-depleted medium. The bacterial dynamics can be investigated by resorting again to the QS approximation. Over brief periods of time the energy remains approximately constant while the speed varies; we thus solve Eq. (1) under the assumptions listed above and the additional approximation $E(t)=E=$ const. For $\xi>2$ we get

$$
v(t)=\left[\frac{E d_{\xi}}{\gamma}+e^{t / T_{\xi}}\left(v_{0}^{2-\xi}-\frac{E d_{\xi}}{\gamma}\right)\right]^{\frac{1}{2-\xi}},
$$



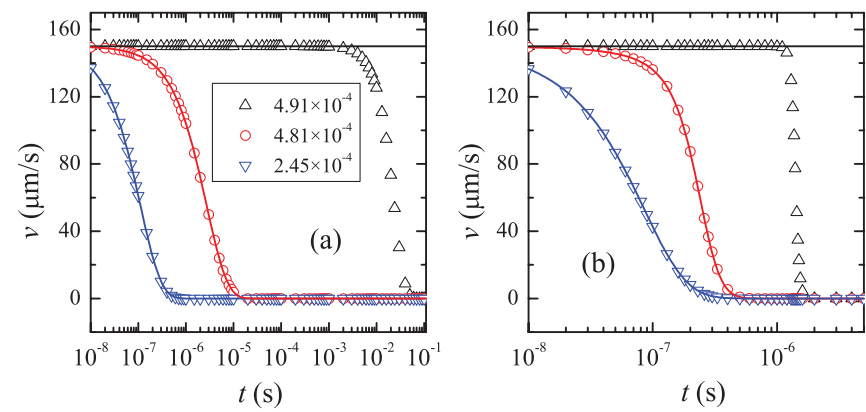

FIG. 3. (Color online) Speed as a function of time for the indicated values of the initial stored energy (erg) for (a) $\xi=2$ and (b) $\xi=3$. The initial speed is $150 \mu \mathrm{m} / \mathrm{s}$ and the rest of the parameters are as described in the caption to Fig. 1. The symbols correspond to simulations of Eqs. (1) and (2) and the solid lines are the analytical approximations, Eqs. (11) and (12). The upward-pointing triangles correspond to the threshold value of the energy for which the QS approximation breaks down. A motor characterized by $\xi \geqslant 2$ would be useless in a depleted medium.

with $T_{\xi}=m /[\gamma(\xi-2)]$, and for $\xi=2$,

$$
v(t)=v_{0} e^{-t / T_{2}}
$$

with $T_{2}=m /\left(d_{2} E-\gamma\right)$. These solutions indicate an abrupt stopping of the bacterium as soon as it enters a nutrientdepleted region, if the starting energy is below the threshold $E_{\xi}^{*}=v_{0}^{2-\xi} \gamma / d_{\xi}$ for $\xi \geqslant 2$. We show in Fig. 3 a comparison between these theoretical predictions and the exact simulations of Eqs. (1) and (2) for the cases $\xi=2$ and $\xi=3$. There is excellent agreement between the simulations and the analytical predictions of Eqs. (11) and (12) except when $E \rightarrow E_{\xi}^{*}$ from below and the QS approximation breaks down. For energies below these thresholds, the bacterial speed drops to zero over a time of the order of $m / \gamma \sim 10^{-6}$ s, i.e., the motion is controlled by the drag force. The slowing down of the true speed, i.e., that obtained from the simulations, when getting close to the threshold is much more pronounced in the case of the crossover exponent $\xi=2$ [19].

If the initial stored energy is larger than the threshold values, both analysis and simulation show that the speed grows very fast while the energy drops rapidly. We therefore conclude that unless nutrients are plentiful everywhere, $\xi \geqslant 2$ motors are inadequate.

The run-and-tumble swimming mode is a characteristic of some peritrichously flagellated bacteria such as E. coli and $S$. typhimurium; it is not the only swimming pattern used by bacteria for space exploration and chemotaxis. Vibrio alginolyticus, for instance, has a single polar flagellum and was known to follow a run-and-reverse motility pattern, the most common search strategy used by marine bacteria [28]. Recently, Xie et al. found that V. alginolyticus actually executes a cyclic, three-step (forward, reverse, and flick) swimming pattern $[29,30]$. The change of direction induced by the flick of the flagellum after a backward run converts the resulting movement pattern into an effective random walk. There are other swimming modes used for space exploration and chemotaxis, such as run-and-stop and run-and-arc [31]. It is easy to see that $\xi=1$ maximizes space exploration independently of the chosen swimming pattern if we consider

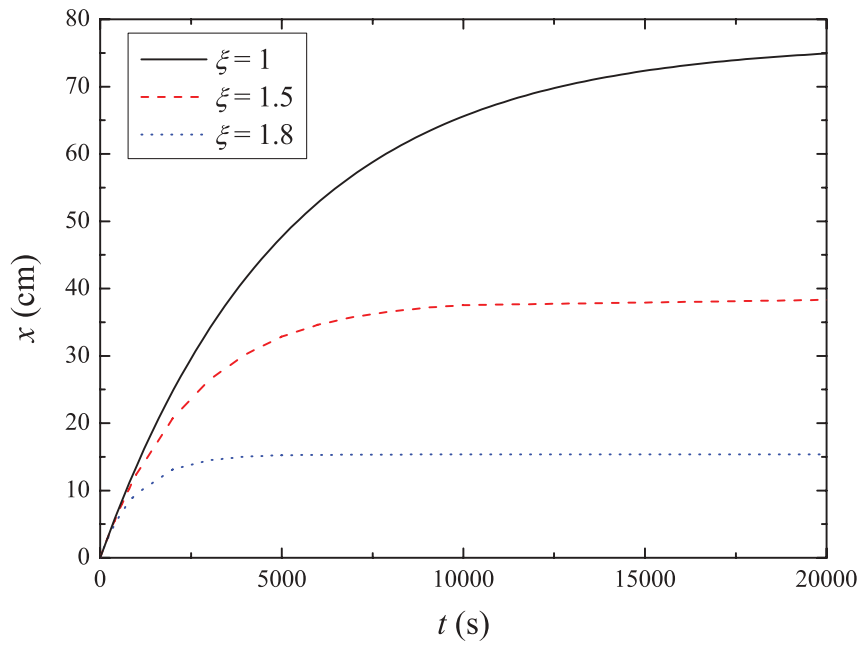

FIG. 4. (Color online) Total linear lengths of an "unscrewed" bacterial trajectory for a bacterium of $a=0.5 \mu \mathrm{m}$ and the values of $\xi$ indicated in the figure. The rest of the parameters are as described in the legend of Fig. 1. The total length is always a monotonically decreasing function of $\xi$. Thus $\xi=1$ optimizes space exploration among all physically acceptable values of $\xi$, independently of the swimming pattern.

the total linear length $x$ of the bacterial path. This length may be obtained by direct numerical integration of Eqs. (1) and (2) and is shown, for a specific bacterial radius, in Fig. 4. These and numerous other numerical results show that in all cases the linear length is a monotonically decreasing function of $\xi$. Consequently, we conclude that $\xi=1$ must be the optimal exploration exponent for arbitrary swimming strategies.

In our study we have neglected the effect of various types of fluctuations. Passive (i.e., thermal) and active fluctuations has been recently studied by Romanczuk and SchimanskyGeier [32], who showed that active fluctuations can have an important influence on microorganism dynamics. It would be interesting to know their effect on space exploration, but we know of no reasons to doubt the robustness of the results presented in this paper.

\section{DISCUSSION}

In this paper, we have extended the analysis of the model discussed in Ref. [19] in order to find the optimal dependence of the power transfer function with microorganism speed. By "optimal" we mean the functional form that maximizes space exploration in a resource-depleted medium such as the ocean. We concluded that regardless of the swimming pattern, space exploration is maximized when the speed exponent in the energy conversion function is near unity, which agrees with experiments that indicate that the torque does not depend on angular speed, except at high $\omega$. We thus conjecture that the independence of torque on angular speed is a trait that some motile bacteria have evolved in order to maximize space exploration in the ocean. We also used realistic values of the relevant parameters to obtain estimates of the size of the explored regions for bacteria that swim following a run-andtumble pattern. Our findings indicate that bacteria for which $\xi$ increases beyond unity are at a competitive disadvantage in 
low nutrient media. Since $\xi<1$ is not allowed, all bacteria should have a value of $\xi$ close to 1 , with little variation. Exceptions may arise if the energetic costs of building the optimal propulsion machinery exceed the benefits of a better space exploration.

Further support for our hypothesis about the evolutionary origin of the plateau region of the torque vs rotational frequency relationship is given by the work of Xing and coworkers [15], who followed a very different line of reasoning to conclude that the plateau corresponds to thermodynamic and Stokes efficiencies of the order of $100 \%$ (the Stokes efficiency is defined as the ratio of the power dissipated by a viscous load to the power used by the motor.) This result was found by assuming that there is a soft elastic linkage between the motor and the load and a separation of time scales between the motor and the load dynamics. In the description of Ref. [15], at high rotation rates the stator dynamics affects the motor torque in such a way that a given stator hinders rotor rotation and the efficiency is decreased. Evidently, the optimal conditions for the bacterium would be for its motors to always work at the plateau that guarantees $100 \%$ thermodynamic efficiency. The same conditions would maximize its search efficiency. A question then arises: Why are there some bacteria, such as V. alginolyticus, that usually do not swim in the constant torque region? We can conjecture that even if evolution has tried to adapt the motor so it maximizes both space exploration and thermodynamic efficiency, this goal has had to compete against the building of what may be an energetically very costly optimal architecture. A compromise may have been to keep a plateau and to make the load line cut the torque-speed curve as close to the knee as possible. Caulobacter crescentus, on the other hand, which swims with a load line cutting the plateau, seems to have reached the perfect compromise [14].

What are the essential elements in the model that lead to our results? We argue that our main conclusion is robust in the sense that we do not need to solve the model to obtain the main result qualitatively. We only need to assume that the power converted into kinetic energy is $P_{K}=k(v) E=d_{\xi} v^{\xi} E$ and that the power converted into all other forms of energy (metabolism, dissipation, etc.) is $P_{A}=c E$. Given a fixed initial amount $E_{0}$ of available energy, we expect that space exploration will be favored if a higher proportion of the stored energy is transformed into kinetic energy. The ratio to calculate is

$$
P_{K} / P_{A}=k(v) / c=d_{\xi} v^{\xi} / c
$$

which, expressed in terms of the dimensionless speed $w=$ $v / v_{0}$, reads

$$
P_{K} / P_{A}=P_{K}^{(0)} / P_{A}^{(0)}\left(v / v_{0}\right)^{\xi}=\Gamma_{0} w^{\xi} .
$$

Here the definition of $\Gamma_{0}$ introduced in Eq. (9) has been used. It depends on the initial speed and available energy and on the friction coefficient $\gamma$, the other key physical parameter in the problem. Since $v / v_{0}<1$ at all times, the power effectively converted into kinetic energy is larger for the smaller values of $\xi$. But $\xi<1$ being forbidden, $\xi=1$ must be optimal, as found from the detailed calculation. This argument can be extended to a general analytical function $k(v)$, provided that $k(0)=0$. Let

$$
k(v)=\sum_{p=1}^{\infty} k_{p} v^{p}
$$

with the initial condition

$$
\frac{k\left(v_{0}\right)}{c}=\sum_{p=1}^{\infty} \frac{k_{p}}{c} v_{0}^{p} \equiv \sum_{p=1}^{\infty} R_{p} .
$$

Hence,

$$
\frac{P_{K}}{P_{A}}=\frac{k(v)}{c}=\sum_{p=1}^{\infty} R_{p} w^{p} .
$$

Since $w<1$, the leading ( $p=1)$ term becomes more and more dominant as $w$ decreases, which qualitatively agrees with the explicit results of the model.

A few additional considerations on the assumptions included in our model are in order: (1) Physics sets strong constraints on the power transfer function. Assuming a powerlaw form for the power transfer function, $k(v) \sim v^{\xi}$, we found that the choice $\xi<1$ is unphysical, because it leads to infinite low-speed torques. Therefore, any functional form for $k(v)$ that grows more slowly than linear at small speeds is also unphysical. Similarly, we can conclude that any power transfer function that grows with a power higher than quadratic cannot describe motion in an oligotrophic medium, although it might be suitable for bacteria that reside in regions of high nutrient concentrations. (2) We have neglected Brownian noise in our formulation, because it would not significantly change the speed of a fast bacterium. The main effect of rotational diffusion would be to somewhat reduce the effective diffusion coefficient, due to run curvature, but this effect is quite independent from the process detailed in this work and would not change our results.

Although the swimming strategy of oceanic bacteria may be strongly dependent on the turbulent environment that they find themselves in, the development of an energetically expensive propulsion system proves that turbulent mixing is insufficient to ensure the efficient meeting of bacteria and nutrients. Turbulent mixing could help define how far a bacterium has to move to find a patch of food. However, even if the advective velocity of the turbulent fluid is of the order of $1 \mathrm{~cm} / \mathrm{s}$, the Reynolds number is of the order of $10^{-2}$. Therefore, bacteria swim in the creeping flow regime, for which the hydrodynamic equations are linear [33]. Thus the effects of turbulence can be considered to be additive. They would affect both the seeking bacteria and the target food patches, but this would not modify our results, which indicate that whatever the search strategy $\xi=1$ is most favorable.

We remark that some results in this paper can be directly tested. For instance, a small increase in the viscosity of the medium (achieved, for instance, through the addition of a small quantity of a viscous agent, such as Ficoll [12]) would result in a variation of the QS speed proportional to $\gamma^{-\psi}$ for all times $t<\tau_{\xi}$, where $\tau_{\xi}$ is the characteristic time for energy storage [19]. This means a much faster decrease in speed with $\gamma$ for higher values of $\xi$. As a consequence, we see from Eq. (7) that 
the radius of the explored region is also predicted to shrink with increasing viscosity as $\eta^{-\psi}$. In particular, if $\xi=1$, we predict that $R \sim v_{Q} \sim \eta^{-1}$ for $t<\tau_{1}=\left[q d_{1}^{2} / \gamma+(c / 2)^{2}\right]^{-1 / 2}$.

The possible generality of the initial constant regime in the torque-frequency relationship in the bacterial transport phase could be tested by direct measurement in other, especially marine bacteria. It should also be experimentally doable to measure possible changes in the correlation torque frequency by placing the bacteria under given nu- tritional conditions for generations-evolution in a test-tube experiment.

\section{ACKNOWLEDGMENTS}

We thank the anonymous reviewers for helpful comments. This work was supported by SECyT-UNC (Project No. 05/B354) and CONICET (PIP 112-200801-00772) (Argentina).
[1] D. W. Menzel and J. H. Ryther, in Symposium on Organic Matter in Natural Waters., edited by D. W. Hood (Inst. Mar. Sci. Univ. Alaska Publ., Fairbanks, Alaska, 1970), Vol. 1, p. 31.

[2] N. Blackburn, T. Fenchel, and J. Mitchell, Science 282, 2254 (1998).

[3] J. G. Mitchell and K. Kogure, FEMS Microbiol. Ecol. 55, 3 (2006).

[4] R. Stocker, J. R. Seymour, A. Samadani, D. E. Hunt, and M. F. Polz, Proc. Natl. Acad. Sci. USA 105, 4209 (2008).

[5] J. A. Novitsky and R. Y. Morita, Marine Biol. 48, 289 (1978).

[6] G. J. Sibona, Phys. Rev. E 76, 011919 (2007).

[7] D. B. Dusenbery, Proc. Natl. Acad. Sci. USA 94, 10949 (1997).

[8] M. D. Manson, P. Tedesco, H. C. Berg, F. M. Harold, and C. van der Drift, Proc. Natl. Acad. Sci. USA 74, 3060 (1977).

[9] N. Hirota and Y. Imae, J. Biol. Chem. 258, 10577 (1983).

[10] H. C. Berg, Annu. Rev. Biochem. 72, 19 (2003).

[11] Y. Sowa and R. M. Berry, Q. Rev. Biophys. 41, 103 (2008).

[12] X. Chen and H. C. Berg, Biophys. J. 78, 1036 (2000).

[13] Y. Sowa, H. Hotta, M. Homma, and A. Ishijima, J. Mol. Biol. 327, 1043 (2003).

[14] G. Li and J. X. Tang, Biophys. J. 91, 2726 (2006).

[15] J. Xing, F. Bai, R. Berry, and G. Oster, Proc. Natl. Acad. Sci. USA 103, 1260 (2006).

[16] T. Mora, H. Yu, and N. S. Wingreen, Phys. Rev. Lett. 103, 248102 (2009).
[17] Y. Magariyama et al., Biophys. J. 69, 2154 (1995).

[18] Y. Magariyama, S. Sugiyama, and S. Kudo, FEMS Microbiol. Lett. 199, 125 (2001).

[19] C. A. Condat and M. E. Di Salvo, Phys. Rev. E 84, 011911 (2011).

[20] F. Schweitzer, W. Ebeling, and B. Tilch, Phys. Rev. Lett. 80, 5044 (1998).

[21] W. Ebeling, F. Schweitzer, and B. Tilch, BioSystems 49, 17 (1999).

[22] C. A. Condat and G. J. Sibona, Physica D 168-169, 235 (2002).

[23] V. Garcia, M. Birbaumer, and F. Schweitzer, Eur. Phys. J. B 82, 235 (2011).

[24] H. C. Berg and E. M. Purcell, Biophys. J. 20, 193 (1977).

[25] B. E. Logan and D. K. Kirchman, Mar. Biol. 111, 175 (1991).

[26] H. C. Berg, E. coli in Motion (Springer, New York, 2004).

[27] J. G. Mitchell, Microb. Ecol. 22, 227 (1991).

[28] S. Kudo, N. Imai, M. Nishitoba, S. Sugiyama, and Y. Magariyama, FEMS Microbiol. Lett. 242, 221 (2005).

[29] L. Xie, T. Altindal, S. Chattopadhyay, and X-L. Wu, Proc. Natl. Acad. Sci. USA 108, 2246 (2011).

[30] R. Stocker, Proc. Natl. Acad. Sci. USA 108, 2635 (2011).

[31] J. G. Mitchell, Am. Nat. 160, 727 (2002).

[32] P. Romanczuk and L. Schimansky-Geier, Phys. Rev. Lett. 106, 230601 (2011).

[33] A. Al-Homoud and M. Hondzo, Appl. Microbiol. Biotechnol. 79, 643 (2008). 